\title{
Does Bereavement-Related Major Depression Differ From Major Depression Associated With Other Stressful Life Events?
}

\author{
Kenneth S. Kendler, M.D., John Myers, M.S., and Sidney Zisook, M.D. \\ Virginia Institute of Psychiatric and Behavioral Genetics and the Departments of Psychiatry and \\ Human Genetics, Medical College of Virginia/Virginia Commonwealth University; and the \\ Department of Psychiatry, University of California at San Diego.
}

\begin{abstract}
Objective: Of the stressful life events influencing risk for major depression, DSM-III and DSMIV assign a special status to bereavement. A depressive episode that is bereavement-related and has clinical features and course characteristic of normal grief is not diagnosed as major depression. This study evaluates the empirical validity of this exclusion criterion.
\end{abstract}

Method: To determine the similarities of bereavement-related depression and depression related to other stressful life events, the authors identified and compared cases on a range of validators in a large-population-based sample of twins. The authors evaluated whether cases of bereavement-related depression that also met DSM criteria for "normal grief" were qualitatively distinct from other depressive cases.

Results: Eighty-two individuals with confirmed bereavement-related depression and 224 with confirmed depression related to other stressful life events were identified. The two groups did not differ in age at onset of major depression, number of prior episodes, duration of index episode, number of endorsed "A criteria," risk for future episodes, pattern of comorbidity, levels of extraversion, risk for major depression in their co-twin, or the proportion meeting criteria for "normal grief." However, individuals with bereavement-related depression were slightly older, and more likely to be female, and had lower levels of neuroticism, treatment-seeking, and guilt and higher levels of fatigue and loss of interest. Interaction analyses failed to find unique features of people whose illness met criteria for both bereavement-related depression and normal grief compared to those whose illness was related to other life stressors.

Conclusions: The similarities between bereavement-related depression and depression related to other stressful life events substantially outweigh their differences. These results question the validity of the bereavement exclusion for the diagnosis of major depression.

Of all the possible stressful life events that could contribute to episode onset for major depression, DSM-III assigned special status to bereavement. If a depressive episode resulted from the death of a loved one and had clinical features consistent with those of normal grief, DSMIII considered it "uncomplicated bereavement," not major depression. None of the other classes of predisposing stressful life events (1-3) were similarly treated; DSM-III contained no categories for uncomplicated "illness-related" or "divorce-related" depression. This diagnostic convention continued, with minor modifications, in subsequent DSM editions.

In taking these steps, DSM-III implied one general and one specific claim. The general claim is that bereavement-related depression differs meaningfully from depression related to other

Address correspondence and reprint requests to Dr. Kendler, Virginia Institute for Psychiatric and Behavioral Genetics, Virginia Commonwealth University Medical School, Box 980126, 800 E. Leigh St., Rm. 1-123, Richmond, VA 23298; kendler@ vcu.edu.. 
stressful life events. The more specific claim is that cases of bereavement-related depression with features indicative of normal grief (e.g., duration of 2 months or less, absence of severe impairment, suicidal ideation, and psychomotor retardation) are sufficiently distinct from all other depressive episodes that only they should not be diagnosed as major depression.

We test the validity of these two claims in a large-population-based sample of twins from the Virginia Twin Study of Psychiatric and Substance Use Disorders (VATSPSUD) (4), which contains over 260,000 person-months of data on risk for a range of stressful life events and onsets of major depression in the last year.

\section{Method}

\section{Sample and Diagnostic Assessments}

Participants in this study derive from two interrelated investigations in Caucasian twin pairs who participated in VATSPSUD (4), a sample ascertained from a population-based register formed from a systematic review of birth certificates in the Commonwealth of Virginia. Female-female (FF) twin pairs, from birth years 1934-1974, became eligible if both members responded to a mailed questionnaire in $1987-1988$, the response rate to which was $\sim 64 \%$. The twins were interviewed four times over 10 years, with cooperation rates ranging from $85 \%$ to 92\%. Male-male and male-female (MMMF) pairs (birth years 1940-1974) were initially ascertained directly from registry records containing all twin births by a telephone interview with a response rate of $72 \%$. The second wave of interviews, conducted up to 5 years later, had a response rate of $83 \%$. The mean (SD) age and years of education of the twins were 36.3 $(\mathrm{SD}=8.2)$ and $14.3(\mathrm{SD}=2.2)$ at the fourth $\mathrm{FF}$ interview and $37.0(\mathrm{SD}=9.1)$ and $13.6(\mathrm{SD}=2.6)$ at the second MMMF interview.

At each interview, the participants provided information on the occurrence of stressful life events in each of 15 categories of individual and network life events in each of the 12 months preceding the interview, plus the month of the interview. The history of major depression was assessed by structured psychiatric interview based on the Structured Clinical Interview for DSM-III-R (SCID) (5). Furthermore, at all interview waves, we assessed the occurrence over the last year of 14 individual symptoms, representing the disaggregated nine "A criteria" for major depression in DSM-III-R (e.g., two items for criterion A4 to assess insomnia and hypersomnia). For each symptom, interviewers probed to ensure that it was due neither to physical illness nor medication. The respondents then aggregated the symptoms reported over the last year into co-occurring syndromes. If a depressive syndrome occurred, respondents were asked when it occurred and the months of its onset and remission. The diagnosis of major depression was made using DSM-IV criteria without the bereavement exclusion.

In these data sets, containing 266,409 person-months of exposure from 9,242 individuals, we first identified subjects with bereavement-related depression. Prior work in the sample indicated that the increase in risk for depression after most stressful life events, including death in relatives, is short-lived and largely restricted to the month of the event (2). Therefore, we identified months in which respondents reported both a death of an immediate family member, friend, or other relative and an onset of major depression. We defined those as suggestive bereavement-related depression. However, for the worst depressive episode in each 1-year period (or the only episode if there was just one), the respondent was asked whether "something happened to make you feel that way" or whether the episode occurred "out of the blue." If they answered "something happened," they described the event. For this report, we defined confirmed bereavement-related depression as the co-occurrence in the same month of an onset of major depression and the report of a death of a family member or friend and the confirmation by the respondent that their depression was due to that death. If multiple episodes of major depression occurred during the past year and the bereavement-related depression was not 
considered the most severe, then the bereavement-related depression did not meet the confirmation criteria, and this patient's illness was excluded from further analysis.

For a comparison group for bereavement-related depression, we selected three classes of stressful life events reflecting a range of relatively severe events that predispose to depressive onsets: divorce/separation (divorce, separation, or breakup of romantic relationship), illness (illness, accident, or other serious health event), and job loss (layoff, fired, or other reason for job loss) (2). Cases of depression related to other stressful life events were selected and confirmed in exactly the manner outlined above for bereavement-related depression.

To further examine the clinical significance of these two types of depressive episodes, we operationalized the DSM-IV "normal grief criterion" as a duration of $\leq$ months and an absence of psychomotor retardation, suicidal ideation, and severe work impairment. We could thus determine whether each depressive episode met these proposed criteria for a normal grief response. Our interviews did not inquire about psychotic symptoms related to bereavement or "morbid preoccupation with worthlessness," the other DSM-IV criteria for normal grief.

Lifetime generalized anxiety disorder was diagnosed using DSM-III-R (6) criteria requiring 1 month's minimum duration. Phobia was diagnosed using an adaptation of DSM-III criteria (7) requiring one or more of 22 unreasonable fears that objectively interfered with the respondent's life. Lifetime alcohol and cannabis abuse and dependence were diagnosed by DSM-IV criteria (8). Adult antisocial personality traits were defined as meeting $\geq 3$ of the DSMIII-R (6) "C criteria" for antisocial personality disorder. Conduct and panic disorder were defined with DSM-III-R criteria, except because of the rarity of fully syndromal panic disorder in this sample (9), to improve power, we allowed up to 30 minutes for symptoms to maximize.

\section{Statistical Analysis}

We first sought to compare the clinical and demographic characteristics of bereavement-related depression versus depression related to other stressful life events. Next, we examined whether the "normal grief criterion" identified a different group of individuals in the bereavementrelated depression group compared to the other stressful life event depression group. Thus, the analysis assessed differences in clinical and demographic characteristics from two main effects: 1) bereavement-related depression versus depression related to other stressful life events and 2) meeting versus not meeting "normal grief criterion") and then their interaction. It is the interaction that captures the essence of the DSM bereavement exclusion criterion-that there is something distinct about depressed individuals who have bereavement-related depression and meet "normal grief criterion," compared to those with depression related to other stressful life events whose clinical course is also consistent with these normal grief criterion. Compared to all other individuals with depressive syndromes, only persons with bereavement-related depression meeting normal grief criterion do not merit a DSM-III or DSM-IV diagnosis of major depression.

Analyses were conducted in SAS (10) using the chi square and t tests and logistic, linear, and Cox proportional hazard regression. Two-tailed $\mathrm{p}$ values were used. For our main effects comparison of bereavement-related depression and depression related to other stressful life events, we used a significance criterion of $p<0.05$. Because of the reduced power when examining interactions (11), we used a significance criterion for these analyses of $\mathrm{p}<0.10$.

\section{Results}

\section{Preliminary Analyses}

We identified 82 individuals meeting our definition of confirmed bereavement-related depression and 224 individuals with confirmed depression related to other stressful life events, 
of whom 167 experienced divorce/separation, 36 illness, and 21 job loss (Table 1). Of those with bereavement-related depression, 23 (28\%) met DSM-IV "normal grief criteria." Of the bereavement-related depression cases who did not meet these criteria, 54\% reported psychomotor retardation, $46 \%$ episode length of $>2$ months, $24 \%$ severe work impairment, and $20 \%$ suicidal ideation. The same "normal grief criteria" identified $55(25 \%)$ of those with depression related to other stressful life events, a nonsignificant difference. The individual specific criteria for "normal grief" also did not differ significantly in frequency in the two depression groups (Table 1).

Given the 266,409 person-months of exposure in our data, the risk per person-month for confirmed bereavement-related depression and for bereavement-related depression meeting criteria for uncomplicated bereavement can be calculated at $( \pm$ SE) $0.031(0.0003) \%$ and 0.009 $(0.0002) \%$, respectively.

\section{Comparison of Bereavement-Related Depression and Depression Related to Other Stressful Life Events}

Individuals with bereavement-related depression were significantly more likely to be female and to be older at their index episode than those with depression related to other stressful life events (Table 1). Therefore, we controlled for age and sex in subsequent analyses. These two groups of subjects differed neither in the duration of their index episode nor in the number of previous episodes (Table 1). Nor did the two groups differ in the proportion that had one or more prior episodes ( $43.9 \%$ versus $49.6 \%)\left(\chi^{2}=0.77, \mathrm{df}=1, \mathrm{p}=0.38\right)$. Subjects in the two depression groups did not differ in the number of endorsed "A criteria" for major depression nor in their endorsement rate for depressed mood (A1), loss of interest (A2), weight or appetite changes (A3), sleep changes (A4), psychomotor changes (A5), or difficulty concentrating (A8). However, individuals with bereavement-related depression reported significantly higher rates of loss of interest (A2) and fatigue (A6) and lower rates of guilt (A7).

Compared to individuals with depression related to other stressful life events, those with bereavement-related depression had significantly lower levels of neuroticism but did not differ in level of extraversion. No significant differences were seen between the two groups in the proportion of cases who had a lifetime history of generalized anxiety disorder, phobia, panic disorder, conduct disorder, adult antisocial personality traits, alcohol abuse or dependence, or cannabis abuse or dependence (Table 1).

After the index episode, the proportion of individuals who had one or more subsequent episodes in our ascertainment frame was similar in our two groups (Table 1). A proportional hazards model also showed no differences in the hazard rate for a subsequent depressive episode in bereavement-related depression and other stressful life event depression subjects $\left(\chi^{2}=0.03\right.$, $\mathrm{df}=1, \mathrm{p}=0.84$ ). After we controlled for age, sex of the co-twin, and zygosity, no significant difference was seen in the risk for lifetime major depression in the co-twins of twins with bereavement-related depression versus depression related to other stressful life events $\left(\chi^{2}=0.27, \mathrm{df}=1, \mathrm{p}=0.60\right)$.

\section{Interaction Analyses}

Do individuals whose episode meets criteria for bereavement-related depression with symptoms and a clinical course consistent with "normal grief" have a unique kind of depressive episode compared to those who have depression related to other stressful life events along with similar grief symptoms and course? Of the 22 comparisons that addressed this question (and for which we could obtain statistical estimates), only two-increased male gender and increased risk for major depression in the co-twin — were significantly different between these two groups (Table 1). 


\section{Discussion}

\section{Differences Between Bereavement-Related Depression and Depression Related to Other Stressful Life Events}

These analyses sought to evaluate the assumption implicit in DSM-III and subsequent DSM editions that bereavement-related depression is qualitatively different from depression related to other stressful life events. The two groups were similar in many important ways, including the duration of the index episode, the frequency of severe impairment, the clinical severity of the episode as reflected in the number of endorsed A criteria, the probability of endorsing six of the nine specific A criteria (including suicidal ideation and changes in appetite, sleep, and concentration), the number of prior depressive episodes, the age at onset of major depression, the pattern of comorbidity with a range of common internalizing and externalizing disorders, the risk for future depressive episodes, and the risk of major depression in their co-twin. Of interest, the symptomatic and duration criteria in DSM-IV for normal grief were met by an equal proportion of cases of bereavement-related depression and depression related to other stressful life events.

Despite these substantial similarities, bereavement-related depression and depression related to other stressful life events differed on seven potential validators. Subjects with bereavementrelated depression were slightly older, more likely to be female, and less likely to seek treatment for the index episode, had lower levels of neuroticism, endorsed more frequently loss of interest and tiredness, and reported much less frequently symptoms of guilt. Given the number of tests performed $(\sim 30)$, however, several of these positive results are likely to result from chance alone.

The older age of the subjects with bereavement-related depression is likely artifactual because the mean age of subjects reporting a "death event" in our entire sample (36.2, SD=9.0) was significantly higher than for those reporting divorce/separation, illness, and job loss (33.1, SD= $9.0 ; \mathrm{t}=13.69, \mathrm{df}=6,930, \mathrm{p}<0.0001)$. We observed a female excess of subjects with bereavementrelated depression. If bereavement-related depression arose solely as a result of the environmental stress of bereavement, the pattern of female preponderance in major depression (12) might be reduced. A number of prior studies have observed similar proportions of men and women in bereavement-related depression (13-16), while other studies reported a modest increase in the proportion of women with bereavement-related major depression (17-20).

The low percentage of individuals with bereavement-related depression who sought treatment is consistent with prior observations that those with the "depressions of widowhood" rarely perceived a need for treatment (21). This may reflect the widely shared conviction that bereaved individuals are "supposed" to be depressed, that such depressions are adaptive (22) and selflimited, and that treatment is unnecessary if not unwise (23).

The findings with neuroticism are of particular interest because this personality trait is a good index of the genetic risk for major depression (24-26). These results suggest that individuals with bereavement-related depression have a lower level of genetic risk than those with depression related to other stressful life events and that their depressive episode arose largely as a result of their bereavement. However, this interpretation is not consistent with several other results. Such a hypothesis would predict that individuals with bereavement-related depression would have had fewer prior depressive episodes, and would have a lower risk of subsequent episodes, lower rates of comorbidity, and a lower risk for major depression in their co-twin. None of these predicted patterns was observed here or in our review of the prior literature (27). Overall, our results are not consistent with the hypothesis that bereavementrelated depression is a more environmentally induced syndrome than depression related to other stressful life events. 
Previous research with the VATSPSUD has demonstrated that different types of life events are related to different depressive symptom profiles (28). Thus, it is not surprising that three of nine "A criteria" for DSM-IV differed in rates of endorsement between the two groups. The increased rate of loss of interest in bereavement-related depression may be related to the effects of "withdrawal from ongoing life"- a core component of grief (29). Since sleep efficiency is impaired in grief, and especially so in grief complicated by major depression (30), it is not surprising that bereavement-related depression is associated with considerable daytime fatigue. That guilt was observed in many more individuals with other stressful life events depression than bereavement-related depression may reflect the active role individuals perceive themselves playing in most life events, whereas the death of a loved one is typically removed from the bereaved's locus of control. Indeed, we recently showed that when depressive symptoms occurred in relation to stressful events, guilt was most common after romantic breakups and least common after bereavement (30).

A low percentage of individuals with bereavement-related depression met criteria for symptoms and a course of illness consistent with "normal grief." Strikingly, the same percentage of individuals with depression related to other stressful life events met these criteria. The depressive features that DSM tells us are characteristic of "normal grief" are as likely to be present in depression related to other stressful life events as in bereavement-related depression.

\section{Interaction Analyses}

Our final analyses examined whether the criteria for "normal grief," when applied to cases of bereavement-related depression, identified a unique subgroup of depressed individuals. Contrary to the predictions of DSM-III, we found the predicted interaction in only two of 22 potential validators - a result consistent with chance expectation. Of these two findings, one (a higher-than-expected proportion of men) is not particularly informative about the DSM bereavement exclusion rule. However, the second finding (a greater-than-expected risk for major depression in co-twins of those meeting criteria for both bereavement-related depression and "normal grief") is contrary to prediction. "Normal grief" in the setting of a bereavementrelated depression would be expected to have a lower, not a higher, familial risk for major depression. These interaction analyses, although not highly powered, provide no support for the special treatment given in DSM for "uncomplicated bereavement."

\section{Comparison With Prior Studies}

Our results can be usefully compared with those obtained in the most similar prior study (20). Using data on lifetime major depression from the National Comorbidity Survey, Wakefield et al. first identified subjects who reported a depressive episode in temporal proximity to bereavement and to other kinds of loss. Using DSM-III-R criteria for uncomplicated bereavement, they divided each group into an "uncomplicated" and "complicated" subgroup. They then compared these four groups on 18 indicators: 12 reflecting features of the index episode (number of depressive symptoms, presence of melancholic features, suicide attempt, and the presence of each of the 9A criteria) and six that describe aspects of their history of lifetime depression (duration of longest episode, ever interfered a lot, ever saw a professional, ever hospitalized, ever received medications, and number of recurrent episodes). Uncomplicated bereavement-triggered and other-loss triggered major depression significantly differed on only two of these 18 variables (higher rates of impairment and lower rates of suicidal ideation in the "other-loss" group). Although not a focus of their article, the authors presented results showing that the bereavement-triggered and other losstriggered complicated subgroups were similar on seven of nine comparison variables for which they were examined, differing only in that the other loss-group had higher levels of recurrence and a less prolonged longest duration. 
Although our results were similar to those obtained by Wakefield et al. in a broad sense-both reports found many more similarities than differences between bereavement-related depression and depression related to other stressful life events—our findings differed in a number of details. Given the many methodological differences between the two studies (e.g., different assessment instruments, and age distributions, lifetime versus last year sampling frames, partly overlapping sets of validators, and our ability to confirm the stressful event-major depression association that was not possible in the National Comorbidity Survey), it would be premature to try to explain all the differences as substantive.

\section{Limitations}

These results should be interpreted in the context of three potentially significant methodological limitations. First, the sample was restricted to white twins born in the Commonwealth of Virginia from 1934 to 1974. These results may not extrapolate to other groups, particularly older groups, in which bereavement events are particularly likely to occur. Second, because the null hypothesis can never be proven, the usefulness of our results are closely related to their statistical power. Despite our large sample of person-months, confirmed bereavement-related depression was an uncommon event. Using Cohen's concept of small, medium, and large effect sizes (31), our first set of analyses-the direct comparison of depression related to bereavement versus other stressful life events-had, for continuous measures such as personality or episode duration, approximately $97 \%$ power to detect medium and $34 \%$ power to detect small effects (32, Table 2.3.5, $\mathrm{N}=120$ ). We expect somewhat less power for tests of semicontinuous variables such as the number of prior or future depressive episodes. For the examination of categorical variables, such as prevalence rates of other disorders or risk of illness in co-twins, we can estimate that our comparisons have approximately $41 \%$ power to detect small effects and over $99 \%$ power to detect medium effects (32, Table 7.3.15). Although we had reasonable power to detect all large and nearly all medium effect size differences between our groups of bereavement-related depression and depression related to other stressful life events, our sample sizes were generally insufficient to be able to detect most small effect size differences between these two groups. Our interaction analyses were undoubtedly less powerful and probably had power only to detect large effect sizes.

Third, by requiring cases of bereavement-related depression to be confirmed by the respondent, we underascertained episodes of bereavement-related depression in subject-years containing multiple episodes. We can, however, estimate the degree of underascertainment. In years with a single depressive episode, $71.4 \%$ of the suggestive subjects with bereavement-related depression were confirmed by the respondent while in years with multiple depressive episodes, only $27.5 \%$ of the suggestive episodes of bereavement-related depression were so confirmed. Assuming that the rate of confirmation in years with single episodes was the same as for years with multiple episodes, we identified $\sim 63 \%$ of all the bereavement-related depression (or 82 of an estimated 131 cases). Thus, the estimated risk for bereavement-related depression in our sample per person-month, corrected for underascertainment, is $\sim 0.049 \%$.

\section{Conclusions}

The similarities of bereavement-related depression and depression related to other stressful life events far outweigh their differences. We were unable to show, as predicted by DSM, that cases of depression meeting criteria for both bereavement-related depression and "normal grief" were unique in any way. On their face, these results argue against the continued use of the "bereavement exclusion rule" in DSM-V. However, despite their overall similar results to ours, Wakefield et al. (20) reached a dramatically different conclusion, suggesting that the bereavement exclusion rule should be extended to all episodes of "uncomplicated," life-eventprecipitated depressive episodes. In fact, neither of these studies provides direct empirical 
information relevant to this challenging question. At a minimum, such a study would compare depressive episodes that were precipitated by life events (i.e., bereavement, divorce, illness, or job loss) versus depressive episodes that were not (i.e., "endogenous," which constituted $12.6 \%$ of episodes in our sample). A superior design would compare "endogenous" depressive episodes, those episodes resulting from severely stressful events (and hence more "understandable" or "normal") and those that arose in the context of more moderately stressful events.

In conclusion, both this study and our recent literature reviews $(27,32)$ suggest that bereavement-related depression is probably similar to other forms of major depression. Bereavement-related depression often is recurrent, genetically influenced, impairing, and treatment responsive. These are all characteristics that are likely to be more associated with major depression than with "normal sadness."

\section{Acknowledgments}

Dr. Zisook has received research support from PamLab and Aspect Medical Systems and has received speaking honoraria from GlaxoSmithKline, Forest Pharmaceuticals, AstraZeneca, and Wyeth-Ayerst Laboratories. The remaining authors report no competing interests.

Supported in part by NIH grants MH-40828, MH-49492, and MH-49671 and the VA San Diego Healthcare System.

\section{References}

1. Kessler RC. The effects of stressful life events on depression. Ann Rev Psychol 1997;48:191-214. [PubMed: 9046559]

2. Kendler KS, Karkowski LM, Prescott CA. Stressful life events and major depression: risk period, longterm contextual threat, and diagnostic specificity. J Nerv Ment Dis 1998;186:661-669. [PubMed: 9824167]

3. Brown, GW.; Harris, TO. Life Events and Illness. New York, Guilford: 1989.

4. Kendler, KS.; Prescott, CA. Genes, Environment, and Psychopathology: Understanding the Causes of Psychiatric and Substance Use Disorders. New York, Guilford: 2006.

5. Spitzer, RL.; Williams, JBW. Structured Clinical Interview for DSM-III-R (SCID). Biometrics Research Department, New York State Psychiatric Institute; New York: 1985.

6. American Psychiatric Association. Diagnostic and Statistical Manual of Mental Disorders. Vol. 3rd ed. revised. American Psychiatric Association; Washington, DC: 1987.

7. American Psychiatric Association. Diagnostic and Statistical Manual of Mental Disorders. Vol. 3rd ed.. American Psychiatric Association; Washington, DC: 1980.

8. American Psychiatric Association. Diagnostic and Statistical Manual of Mental Disorders. Vol. 4th ed.. American Psychiatric Association; Washington, DC: 1994.

9. Kendler KS, Gardner CO, Prescott CA. Panic syndromes in a population-based sample of male and female twins. Psychol Med 2001;31:989-1000. [PubMed: 11513384]

10. SAS Publishing. SAS/STAT User's Guide, Version 8. STATS Publishing; Cary, NC: 2000.

11. Wahlsten D. Insensitivity of the analysis of variance to heredity-environment interaction. Behav Brain Sci 1990;13:109-161.

12. Bebbington PE. Sex and depression. Psychol Med 1998;28:1-8. [PubMed: 9483678]

13. Clayton PJ, Halikes JA, Maurice WL. The bereavement of the widowed. Dis Nerv Syst 1971;32:597604. [PubMed: 5112136]

14. Lund DA, Caserta MS, Dimond MF. Gender differences through 2 years of bereavement among the elderly. Gerontologist 1986;26:314-320. [PubMed: 3721240]

15. McHorney CA, Mor V. Predictors of bereavement depression and its health-services consequences. Med Care 1988;26:882-893. [PubMed: 3419233]

16. Zisook S, Shuchter SR. Uncomplicated bereavement. J Clin Psychiatry 1993;54:365-372. [PubMed: 8262878] 
17. Gallagher EG, Breckenridge JN, Thompson LW, Peterson JA. Effects of bereavement on indicators of mental health in elderly widows and widowers. Gerontology 1983;38:565-571.

18. Jacobs S, Hansen F, Berkman L, Kasl S, Ostfeld A. Depressions of bereavement. Compr Psychiatry 1989;30:218-224. [PubMed: 2731419]

19. Bruce ML, Kim K, Leaf PJ, Jacobs S. Depressive episodes and dysphoria resulting from conjugal bereavement in a prospective community sample. Am J Psychiatry 1990;147:608-611. [PubMed: 2327488]

20. Wakefield JC, Schmitz MF, First MB, Horwitz AV. Extending the bereavement exclusion for major depression to other losses: evidence from the National Comorbidity Survey. Arch Gen Psychiatry 2007;64:433-440. [PubMed: 17404120]

21. Clayton PJ, Maurice WL, Halikas JA. Depression of widowhood. Br J Psychiatry 1972;120:71-77. [PubMed: 5041522]

22. Nesse, RM. An evolutionary framework for understanding grief, in Late Life Widowhood in the United States. In: Carr, DS.; Nesse, RM.; Wortman, CB., editors. Spousal Bereavement in Late Life. Springer Publishing; New York: 2005. p. 195-226.

23. Horowitz AV. Distinguishing distress from disorder as psychological outcomes of stressful social arrangements. Health: An Interdisciplinary Journal for the Social Study of Health, Illness and Medicine 2007;11:273-289.

24. Kendler KS, Neale MC, Kessler RC, Heath AC, Eaves LJ. A longitudinal twin study of personality and major depression in women. Arch Gen Psychiatry 1993;50:853-862. [PubMed: 8215811]

25. Fanous AH, Neale MC, Aggen SH, Kendler KS. A longitudinal study of personality and major depression in a population-based sample of male twins. Psychol Med 2007;37:1163-1172. [PubMed: 17407614]

26. Kendler KS, Gatz M, Gardner C, Pedersen NL. Personality and major depression: a Swedish longitudinal, population-based twin study. Arch Gen Psychiatry 2006;63:1113-1120. [PubMed: 17015813]

27. Zisook S, Kendler KS. Is bereavement-related depression different than non-bereavement-related depression? Psychol Med 2007;37:779-794. [PubMed: 17306046]

28. Keller MC, Neale MC, Kendler KS. Association of different adverse life events with distinct patterns of depressive symptoms. Am J Psychiatry 2007;164:1521-1529. [PubMed: 17898343]correction, 165:401

29. Bowlby, J. Attachment and Loss, Volume 3: Loss. Basic Books; New York: 1980.

30. Germain A, Caroff K, Buysse DJ, Shear MK. Sleep quality in complicated grief. J Traumatic Stress 2005;18:343-346.

31. Cohen, J. Statistical Power Analysis for the Behavioral Sciences. Academic Press; Orlando, Fla: 1977.

32. Zisook S, Shear K, Kendler KS. Validity of the bereavement exclusion criterion for the diagnosis of major depressive episode. World Psychiatry 2007;6:38-43. [PubMed: 17342225] 
Kendler et al.

Page 10

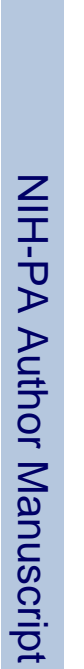

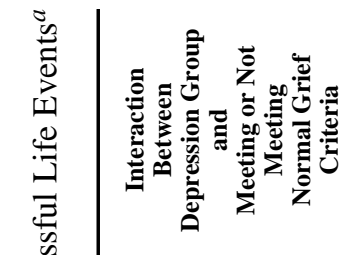

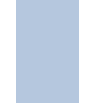

乙

率

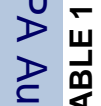

容

.

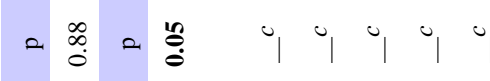

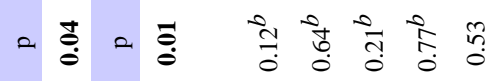

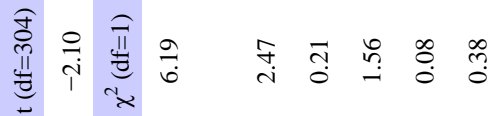

के क्ष

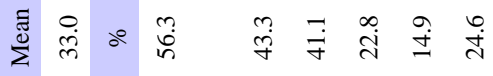

के

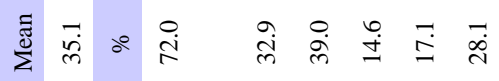

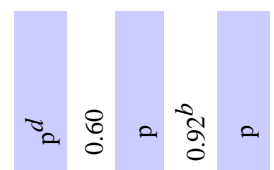

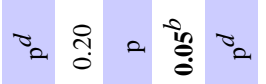

3. $=\frac{5}{5}$ है

$=0$ อ

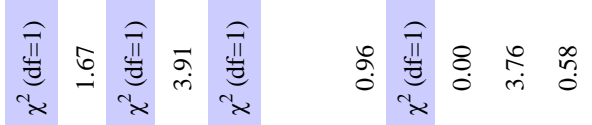

की

कิ

$\exists$

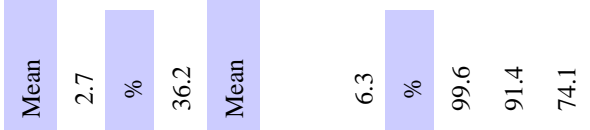

के पं की

突 
Kendler et al.

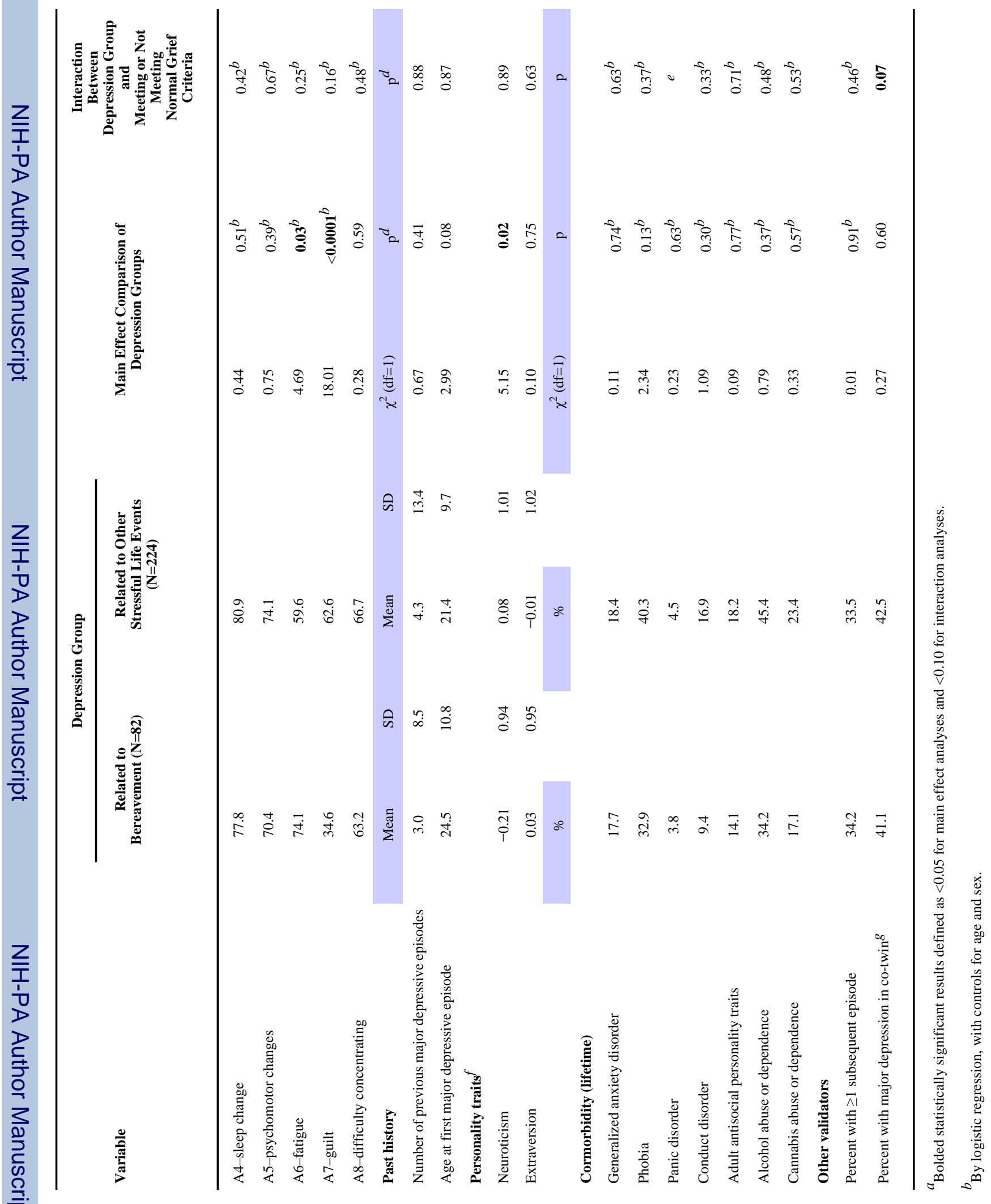
Am J Psychiatry. Author manuscript; available in PMC 2009 November 1. 


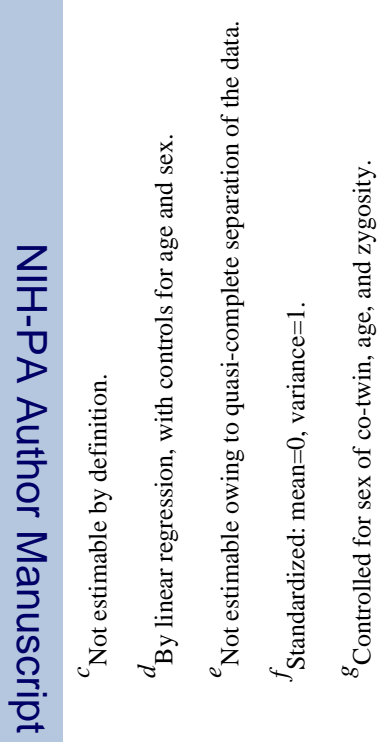

